\title{
MIDEX Advanced Modular and Distributed Spacecraft Avionics Architecture
}

\author{
John A. Ruffa \\ NASA/Goddard Space Flight Center/Code 735 \\ Greenbelt, MD 20771 \\ (301) 286-8247 \\ john.ruffa@gsfc.nasa.gov
}

\author{
Karen Castell \\ NASA/GSFC/Code 734 \\ Greenbelt, MD 20771 \\ (301) 286-2383 \\ karen.d.castell.1@gsfc.nasa.gov
}

\author{
Thomas Flatley \\ NASA/GSFC/Code 733 \\ Greenbelt, MD 20771 \\ (301) 284-7029 \\ thomas.p.flatley.1@gsfc.nasa.gov \\ Michael Lin \\ NASA/GSFC/Code 712 \\ Greenbelt, MD 20771 \\ (301) 286-3835
}

\begin{abstract}
MIDEX (Medium Class Explorer) is the newest line in NASA's Explorer spacecraft development program. As part of the MIDEX charter, the MIDEX spacecraft development team has developed a new modular, distributed, and scaleable spacecraft architecture that pioneers new spaceflight technologies and implementation approaches, all designed to reduce overall spacecraft cost while increasing overall functional capability. This resultant "plug and play" system dramatically decreases the complexity and duration of spacecraft integration and test, providing a basic framework that supports spacecraft modularity and scalability for missions of varying size and complexity. Together, these subsystems form a modular, flexible avionics suite that can be modified and expanded to support low-end and very high-end mission requirements with a minimum of redesign, as well as allowing a smooth, continuous infusion of new technologies as they are developed without redesigning the system. This overall approach has the net benefit of allowing a greater portion of the overall mission budget to be allocated to mission science instead of a spacecraft bus. The MIDEX scaleable architecture is currently being manufactured and tested for use on the Microwave Anisotropy Probe (MAP), an inhouse program at GSFC.
\end{abstract}

\section{TABLE OF CONTENTS}

1. INTRODUCTION

2. MIDEX ARCHITECTURE OVERVIEW

3. MIDEX ENABLING TECHNOLOGIES

4. MIDEX ARCHITECTURE ADVANTAGES

5. CONCLUSION

\section{INTRODUCTION}

MIDEX (Medium Class Explorer) is the newest line in NASA's Explorer spacecraft development program. Developed and managed out of Goddard Space Flight Center (GSFC), the program's goal is to pursue "high-value" space science and astrophysics missions. In addition, the MIDEX program objectives include development and infusion of new technologies, architectures, and operations concepts which increase mission value by lowering cost and increasing capability. There is an emphasis on pioneering "high-risk" technologies via partnerships and collaborations with industry with the ultimate goal of transferring these technologies to industry for commercial spacecraft applications.

It became clear at the beginning of the program that in order to accomplish these goals, significant changes would need to be made in the way that the MIDEX spacecraft hardware was designed and developed. Particular emphasis was paid to the spacecraft architecture itself and how lessons learned from previous GSFC spacecraft development efforts could be used in its development. This resultant "enabling architecture" would need to provide a flexible science platform that readily accommodated the increasing demands that science instruments place on spacecraft performance and operation, but at a fraction of the cost allotted on previous missions. The spacecraft architecture would need to be flexible, allowing high-end and low-end missions of wide ranging complexity to be configured from a single core design. The architecture would focus on using accepted aerospace industry standards and formats to reduce complexity, development time and cost, but still had to easily incorporate non-standard components and instrument platforms with minimal additional cost and development time. In addition, this architecture had to continue to outpace obsolescence in a world of exploding technology development and innovation. This could only be accomplished by allowing a clear path for rapid insertion of new, emerging technologies with minimal non-recurring expense to meet growing science requirements while continuing to reduce development and operating costs.

By incorporating all of these derived requirements as a guide, a study team at GSFC was able to build upon the lessons learned from recent GSFC spacecraft in-house development efforts[1] and capitalize on recent technology 
innovations (including a significant number of GSFC/industry joint technology development partnerships) to develop an advanced architecture concept for the MIDEX spacecraft development program. This architecture incorporates all of the original goals of the MIDEX development effort and falls within the cost and development restrictions placed by the MIDEX program. The first flight design, fabrication, test, and integration of the MIDEX spacecraft architecture is currently being manufactured and tested for use on the Microwave Anisotropy Probe (MAP), an in-house MIDEX spacecraft development program at GSFC. In addition, the architecture concept has been shown to be flexible and robust enough to meet a wide range of spacecraft requirements, a point apparently proven by that fact that since the introduction of the MIDEX spacecraft architecture at least two other NASA missions have adopted all or part of the MIDEX architecture and/or avionics units for use on their missions. In fact, various subsystems in the MIDEX architecture have been successfully commercialized under a NASA Space Act Agreement with Litton Amecom Division, which is now marketing these subsystem components for space applications. A significant number of commercial satellite vendors are in the process of studying the applicability of the MIDEX architecture and subsystem components to their spacecraft implementation requirements.

\section{MIDEX ARCHITECTURE OVERVIEW}

A block diagram of the MIDEX scaleable architecture is shown in Figure 1. As part of the simplified interfaces designed into the architecture implementation, the primary interfaces to each of the subsystem electronics consists of the Dual-Rate 1773 fiber optic bus and spacecraft power.

The MIDEX avionics units consist of the Power System Electronics (PSE) and the MIDEX Attitude Control Electronics (ACE)/Command and Data Handling (C\&DH) unit (MAC). Both of these subsystems follow a similar distributed, modular design concept in their implementation approach. The subsystems utilize common cards sizes, enclosures, and formats and share a common Low Voltage Power Converter design which converts the Spacecraft power bus voltage down to the appropriate voltage levels for each of the subsystem applications. Each of the avionics subsystems utilize an expandable "building block" approach in their designs, coupled with a varied contingent of card types/configurations that can be inserted into the unit to provide the various capabilities required for a specific mission. In addition, each of the subsystems (and the instrument as well) utilize a Remote Service Node (RSN) design that allows them to interface all subsystem components to the spacecraft bus over the 1773 fiber optic interface, perform all subsystem-level commanding and telemetry collection, and utilize standardized software. The implementation of the RSN design into the MIDEX architecture simplifies testing and dramatically reduces integration time at both the spacecraft and subsystem levels. Finally, the modular design of the avionics was specifically engineered to allow a smooth, continuous infusion of new technologies as they are developed without redesigning the system. Together, these subsystems form a modular, flexible avionics suite that can be modified and expanded to support low-end and very high-end mission requirements with a minimum of redesign. Figure 1 illustrates this "highend, low-end" configuration approach by indicating the minimum hardware per subsystem required for a simple spacecraft implementation. At the same time, the MIDEX subsystems have been designed in a modular, scaleable fashion to expand in functional capability by adding cards to the subsystem implementation.

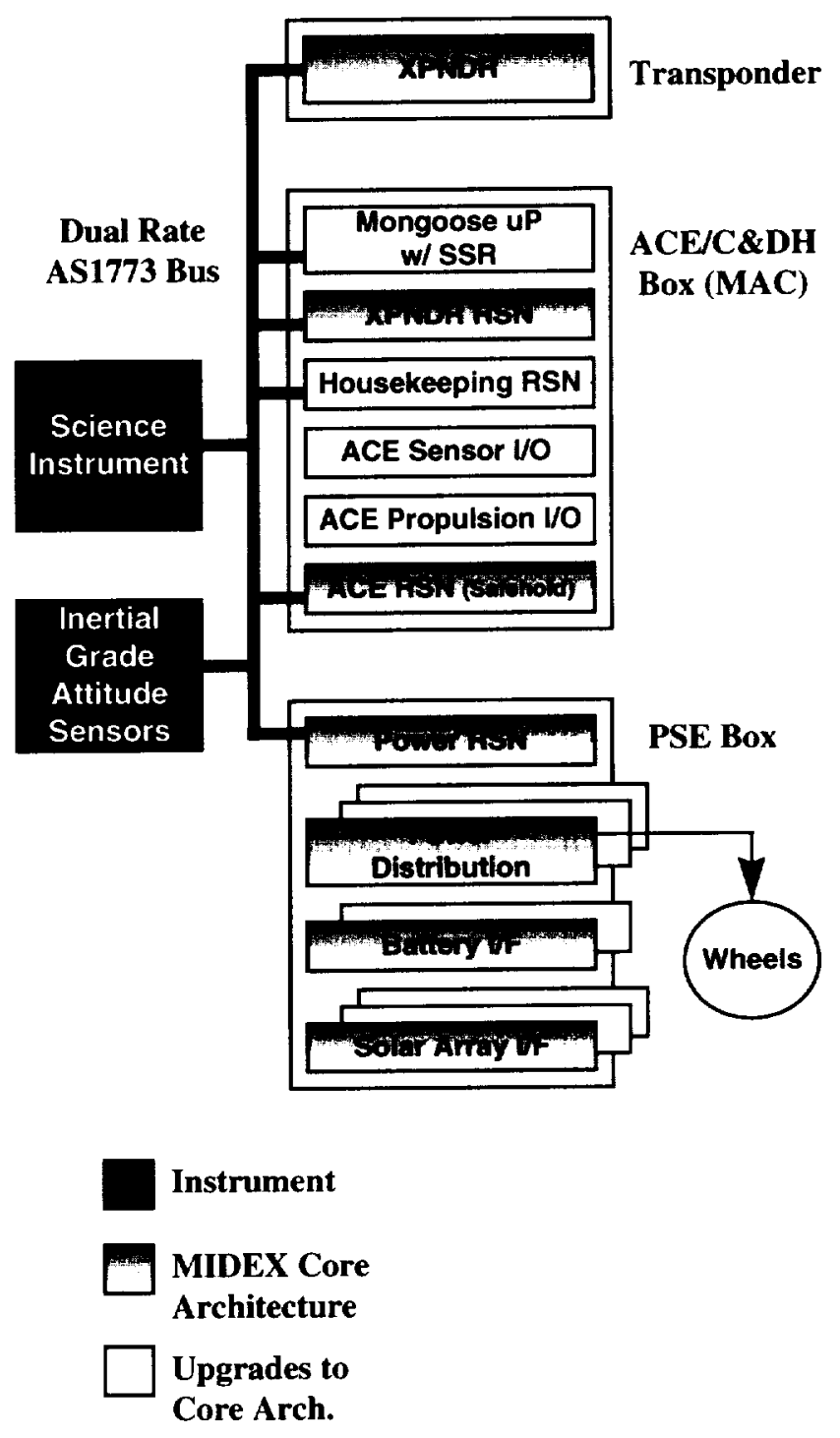

Figure 1 MIDEX Architecture Block Diagram

The following is a functional description of the MIDEX Spacecraft architecture. Although the baseline implementation shown here illustrates a single string (nonredundant) implementation, the actual architecture implementation can be readily modified to incorporate various levels of redundancy with minimal impact. In the 
MAP spacecraft implementation of the MIDEX architecture, the project was initially directed to pursue a single-string spacecraft architecture design approach. However, a move was later made to modify the MAP flight architecture to incorporate redundancy late in the development effort. This change was made in response to suggestions by an independent review team to increase the MAP mission's overall chances of success in light of the tremendous value of the anticipated science and the growing interest from the world astrophysics community. As a result of these recommendations, changes were made to the MAP architectural implementation late in the program at a relatively minor cost impact and virtually no schedule impact, again demonstrating the flexibility and value of the MIDEX architecture.

\section{AS1773 Fiber Optic Data Bus}

The AS1773 fiber optic bus is a key enabling technology for the MIDEX architecture. As previously mentioned, the AS 1773 bus performs as a simple, standardized, inherently redundant command and data interface throughout the spacecraft. In addition, it provides many other benefits such as higher data rates, EMI isolation, and lower overall mass and power consumption than other comparable implementation approaches. The AS1773 implementation will allow dual data rates of $1 \mathrm{Mbps}$ and $20 \mathrm{Mbps}$ between the MIDEX spacecraft components (although the MAP spacecraft will initially implement only the 1 Mbps data rate capability). The AS1773 bus is a continuation of GSFC's ongoing use and experience with time-multiplexed fiber optic data busses in spaceflight applications, including the Solar Anomalous Magnetospheric Particle Explorer (SAMPEX) (launched in 1992)[2], X-Ray Timing Explorer (XTE) (1995)[3], Hubble Space Telescope (HST) Solid State Recorder (SSR) (1997) and the Tropical Rainfall Measurement Mission (TRMM) (1997).

\section{Remote Services Node (RSN) Concept}

At the core of the MIDEX architecture and its modular distributed approach is the implementation of the Remote Service Node (RSN) concept. The RSN concept is based around the Essential Services Node (ESN) Multi-Chip Module (MCM), which acts as a low cost, rad-hard, processor-controlled flexible interface device[4-5]. By implementing RSN technology throughout the spacecraft, all spacecraft subsystem commanding and telemetry collection can be processed at the subsystem level in the individual RSNs. In addition, the RSN implementation also allows the subsystems to be configured in such a way so as to make the 1773 bus and power the primary subsystem interfaces to the spacecraft. These simple, standard interfaces significantly simplifies complexity and decreases the time required for the integration and test of the subsystem with the rest of the spacecraft

Figure 2 illustrates the method by which the ESN is implemented in spacecraft subsystems and components as a standard interface component. The custom interfaces which previously required complex interfaces at the spacecraft level are now relegated to the subsystem level, allowing them to be tested and verified during subsystem-level testing. As a result, the primary interfaces at the spacecraft level are the 1773 fiber optic bus and power, which greatly simplifies the spacecraft interfaces as well as dramatically reducing spacecraft testing and integration. In addition spacecraft power distribution is now distributed to the various spacecraft subsystem components, which switch them via a solid-state circuit breaker under RSN control.

The result is a spacecraft avionics suite that is modular, distributed, equipped with simple, standardized interfaces that facilitate more comprehensive subsystem

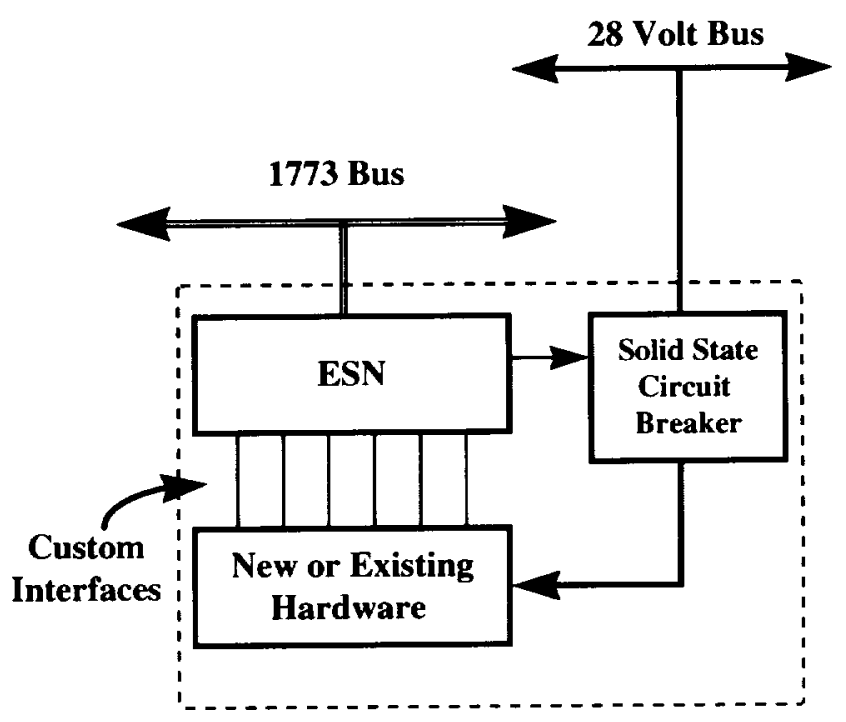

Figure 2 ESN Implementation in the MIDEX Architecture

level testing and simplified spacecraft level integration and test. In addition, non-standard components can be easily integrated into the MIDEX architecture with a minimum of additional design by the addition of the ESN as a standard interface component.

The use of the ESN MCM in the RSN designs also allows the additional benefit of enabling software standardization throughout all of the RSNs in the spacecraft. In the MAP implementation, for example, the flight avionics development team developed a Generic RSN Core design common to all of the RSNs on the spacecraft. This standard RSN design implementation (known as the Generic RSN Core) encapsulates all of the basic design requirements that were required in the MIDEX architecture implementation.

The Generic RSN core design covers approximately half of one side of a 9.7" $\times$ 8.4" MAP standard flight surface-mount card, leaving the remaining half-side as well as the alternate side of the flight card for the designer to implement the application specific design required for the specific 
subsystem application. The diagram in Figure 3 illustrates this implementation approach, as well as identifying the standard backplane and faceplate connectors

used by the Generic RSN Core design. This implementation approach results in a tremendous of amount of hardware and software commonality between subsystem RSN implementations on the spacecraft. In fact, with the exception of the Attitude Control Electronics (ACE) RSN (which implements an independent safehold capability and, therefore, has a significantly larger application-specific code usage), the RSNs in each of the spacecraft and instrument subsystems have a common flight software code use of between 57\% (Instrument RSN) and $81 \%$ (XRSN) of total flight code per card. This commonality in hardware and software simplifies both hardware and software interfaces, once again allowing increased capability at a lower cost.

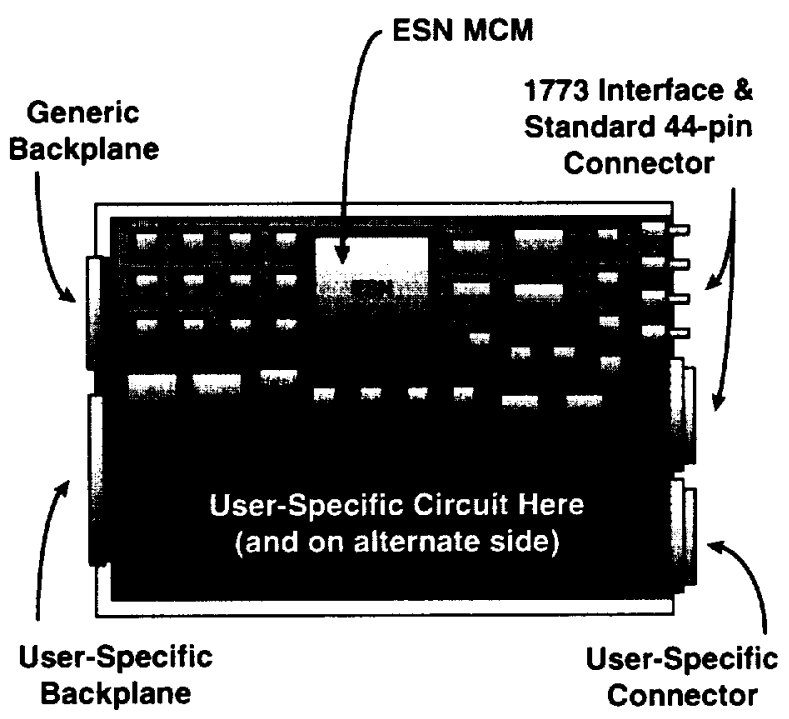

Figure 3 Generic RSN Core Implementation Approach

The Generic RSN Core design also has a standard interface to the standard LVPC card designs used in the MIDEX architecture. Through this command interface, each RSN controls the LVPC switched power outputs by communicating over a standard backplane design. These LVPC switched power outputs are, in turn, used to provide power to subsystem components. In addition, the RSN/LVPC combination is used to provide autonomous spacecraft heater control for each subsystem, with the RSN reading subsystems thermistor telemetry and switching on and of subsystem heaters via the LVPC in order to maintain the desired thermal environment.

The actual Generic RSN hardware implementation on the MIDEX standard 9.7" $\times$ 8.4" flight card size is shown in Figure 4.

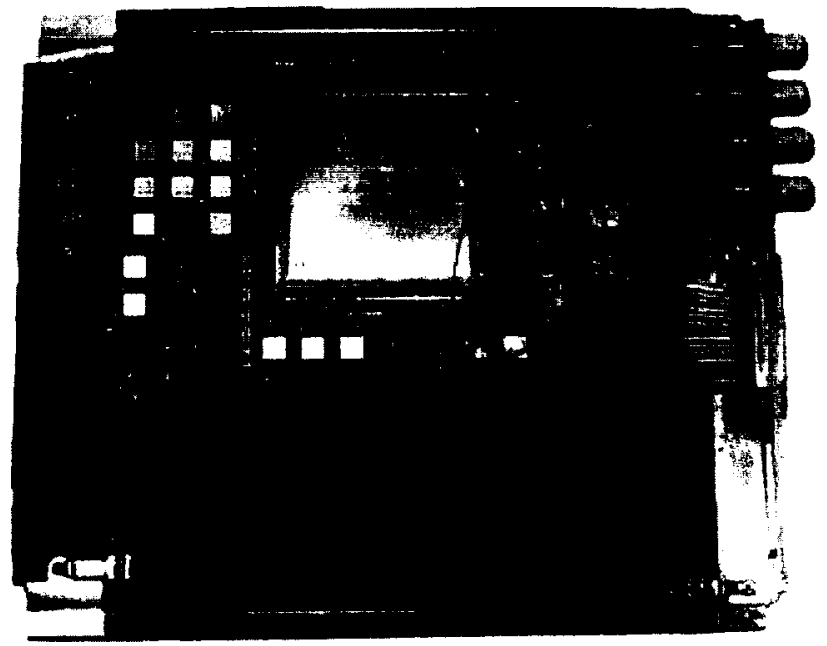

Figure 4 Actual Generic RSN Hardware Implementation

\section{Power Supply Electronics (PSE)}

The guiding principles for the MIDEX PSE subsystem design were similar to those of the rest of the MIDEX spacecraft architecture: that the PSE design must readily apply to the implementation requirements of multiple missions. In practical terms, this meant that the PSE design must be able to accommodate the following requirements: the external interfaces must be simplified and standardized to reduce the costs of subsystem and spacecraft level test and integration, and the PSE must be designed to allow the use of cost reducing technology advances in solar arrays and batteries. In addition, the PSE design must have a modular power output implementation approach to support baseline missions with up to $400 \mathrm{~W}$ orbital average loads in LEO orbits with solar arrays oriented towards the sun, as well as the ability to accommodate higher power missions with minimal size, weight, and cost growth.

The PSE subsystem consists of the following hardware components: the PSE RSN, Power Distribution Modules, Battery Interface Modules, and Solar Array Interface Modules.

PSE RSN Card-The PSE RSN (shown in Figure 5) is the central processor in the PSE electronics. Based around the Generic RSN Core electronics, this card provides digital control of all of the PSE functions and modules. A number of control algorithms can be implemented in the PSE RSN to adapt to a wide variety of spacecraft mission requirements and PSE configurations. The RSN provides the power system with autonomous control-all power system functions and fault detection and correction can be managed within the subsystem. In addition, having the power system control algorithm accomplished in table-driven software allows for flexibility in power system component interfacing. Any battery technology or solar array size can be accommodated by adjusting calibration constants and signal gains in the software and adjusting jumpers on the interface boards. The system transient response to load changes can be tuned to various mission power profiles by simply modifying software control factors. 


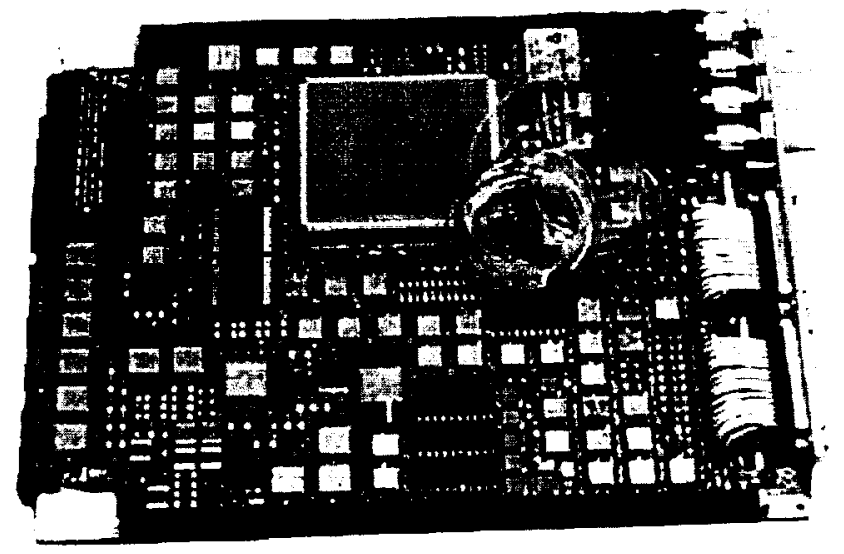

Figure 5 PSE RSN Card

Power Distribution Modules- The PSE Power Distribution Modules provide both switched and unswitched power to the various spacecraft subsystems. The modular design of the PSE unit supports the capability to hold up to three power distribution modules, depending upon the power requirements and configuration of the selected spacecraft.

This is the total number of Power Distribution Modules that can be implemented in the baseline PSE design configuration without forcing additional design and cost The Power Distribution Modules rely entirely on solid state power switching technology (instead of relays), the status of which is constantly monitored by the PSE RSN. These solid state switches provide resettable overcurrent protection of the loads without fuses.

Battery Interface Modules- The Battery Interface Module provides the electrical interface by which the spacecraft batteries provide power to the spacecraft or are charged and conditioned via the spacecraft solar arrays. The modular design of the PSE allows the use of up to two Battery Interface Modules (once again, without incurring redesign and cost), depending upon the number and size of the batteries required for the particular spacecraft. Any battery technology ( $\mathrm{NiCd}, \mathrm{NiH} 2, \mathrm{Li}$ Ion) can be incorporated with minor adjustments in software and firmware. Battery sizes up to $50 \mathrm{Amp}$-hours per module can be used.

Solar Array Interface Modules- These modules provide the interface between the PSE and the spacecraft solar arrays. Up to three Solar Array Modules can be implemented in a modular fashion in order to configure the PSE subsystem to meet the solar array power generation requirements dictated by the spacecraft. The Solar Array Interface Modules are adaptable to multiple solar array sizes of up to 750 Watt per card by installing jumpers and modifying software constants. Power dissipating solar array shunts have been replaced with rad-hard, solid state, nondissipating FETs (Field Effect Transistors) with low on resistance.

\section{Command \& Data Handling ( $C \& D H)$}

The MIDEX C\&DH system (which is housed in a common enclosure with the ACE subsystem electronics) functions as the sole interface between the MAP spacecraft/instrument subsystems and the ground operations equipment. The C\&DH system acts as a command decoding and distribution system, a telemetry/data handling system, and a data storage and playback system. It provides on-board computational capability for processing attitude sensor data and generating commands for the attitude control actuators in a closed-loop fashion. It also provides stored command processing and monitoring of the health and safety functions for the spacecraft and instrument subsystems.

The C\&DH consists of the following hardware components: the Mongoose V Spacecraft Processor and Solid State Recorder (SSR) Card, Transponder RSN Card, and Housekeeping RSN card.

Mongoose $V$ Spacecraft Processor/SSR Card- The Mongoose V 32-bit Rad-hard RISC Processor card (shown in Figure 6) acts as the spacecraft central processor in the MAP spacecraft implementation and is responsible for the bulk of the spacecraft processing capability. The Mongoose $\mathrm{V}$ processor card also acts at the Bus controller $(\mathrm{BC})$ on the Spacecraft 1773 fiber optic bus. The processor card also includes 320 Mbytes of on-board stacked DRAM used for processor local memory and spacecraft SSR. This DRAM is configured in the following manner: 256 Mbytes (64M x 32) of actual data storage and 64 Mbytes $(64 \mathrm{M} \times 8)$ of DRAM dedicated to Error Detection \& Correction (EDAC).

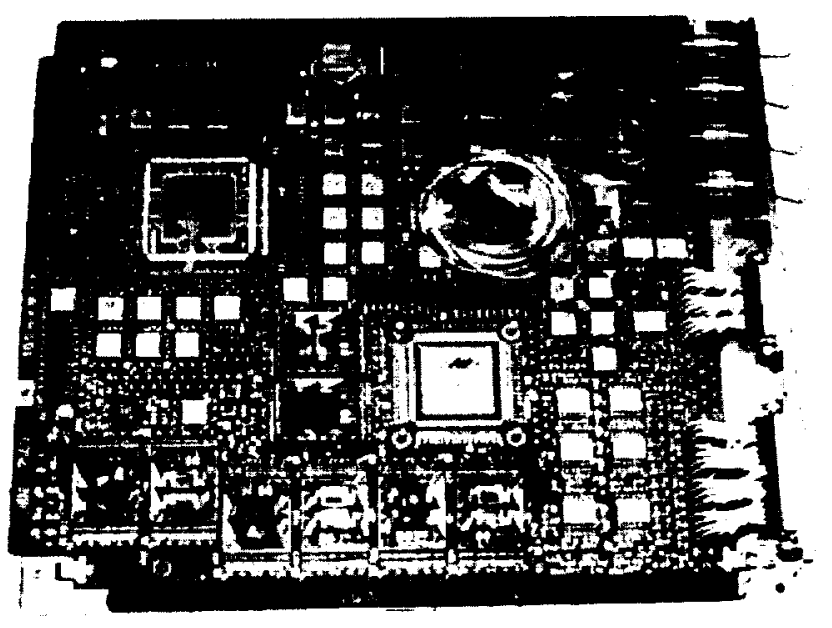

Figure 6 Mongoose V Processor/SSR Card

In addition, the Mongoose V Processor card also includes the following capabilities:

- 4 Mbytes of jumper-configurable EEPROM, divided into protected, write-protected, and flight-writeable areas;

-Spacecraft Time Keeper, which maintains Mission Elapsed Time in seconds and sub-seconds;

-Watchdog Timer Circuit, which provides a timed reset capability;

-External Timer, which provides a timed interrupt capability; 
-Medium Speed Serial Port (MSSP) to transfer data serially to the MAP Transponder RSN (XRSN) via an RS422 interface;

-Hardware Data Compression option;

-External Waitstate Generators.

Transponder RSN (XRSN)- The XRSN (shown in Figure 7) provides the Command and telemetry link between the C\&DH subsystem and the ground system. Its is based around the Essential Services Node (ESN) Multi-Chip Module (MCM) and uses a modified version of the Generic RSN design and layout. The XRSN shall receives CCSDS (Consultative Committee for Space Data Systems) uplink codeblocks from the transponder and passes them to the C\&DH. The XRSN also receives CCSDS downlink frames from the C\&DH, formats and encodes them, and sends them to the transponder for transmission to the ground system. The maximum Uplink data rate possible with the XRSN is $10 \mathrm{Kbits} / \mathrm{sec}$. The maximum transfer rate (data and encoding) from the XRSN to the GN transponder (which is used in the MAP spacecraft implementation) for downlink telemetry is $6 \mathrm{Msymbols} / \mathrm{second}$.

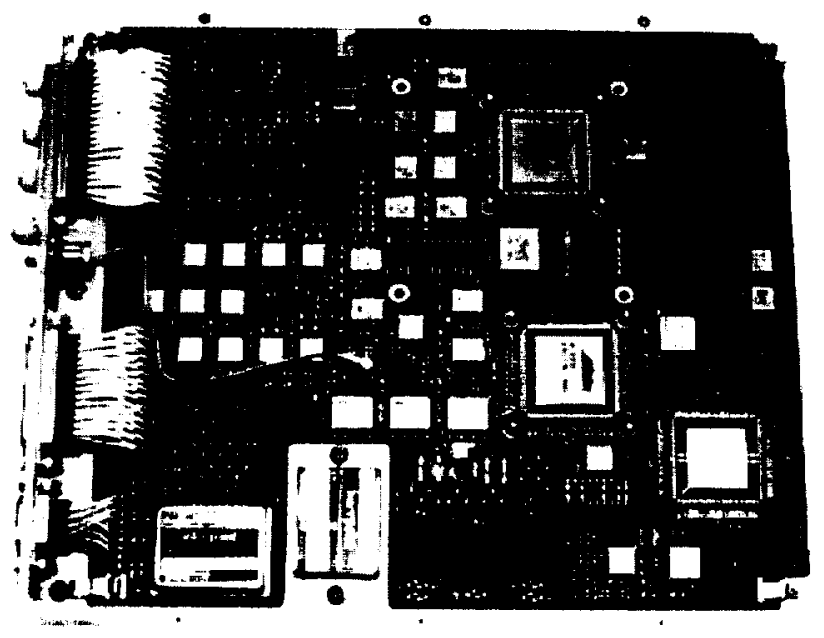

Figure 7 XRSN card (showing alternate side of card)

The XRSN interfaces with the C\&DH via a serial $\mathrm{RS} 422$ telemetry interface and the 1773 bus. The High-Rate Telemetry interface provides the XRSN with high-rate telemetry frames to downlink. The 1773 bus provides the XRSN with the following: XRSN commands, GN transponder commands, low-rate telemetry frames to downlink, and time-code updates. The 1773 bus provides the C\&DH with the following: uplink codeblocks, GN transponder housekeeping telemetry, and XRSN housekeeping telemetry.

The XRSN has the capability to decode and distribute up to eight (8) special hardware commands. These discrete command signals (hardware decoded discrete commands wired from the XRSN to the affected subsystem interface) are used to change the configuration of the MAP spacecraft independent of the 1773 bus.
For the MAP spacecraft implementation, the XRSN is designed to interface to either the Loral Ground Network (GN) transponder or the Fourth Generation TDRSS Transponder. If interfacing to the GN transponder, the XRSN shall interface the transponder to the C\&DH via the 1773 bus, controlling the transponder mode and sampling transponder status.

Housekeeping RSN (HRSN)- The Housekeeping RSN (HRSN) is responsible for two primary functions. First, it performs all of the $\mathrm{C} \& \mathrm{DH}$ subsystem-level housekeeping telemetry collection and discrete commanding associated with the subsystem. Second, it performs MAP spacecraft level housekeeping telemetry collection and discrete commanding associated with various spacecraft components not associated with another subsystem.

The HRSN design is based on the Generic RSN Core design and layout. The HRSN builds upon this design to provide housekeeping telemetry collection and discrete commanding functions to the C\&DH subsystem and the MAP Spacecraft. This command and telemetry collection capability interfaces to the MAP architecture via a MIL-STD-1773 bus.

As an example of the HRSN implementation and use, in the MAP spacecraft implementation the HRSN performs the following functions:

-Spacecraft Thermistor \& Voltage Monitoring;

-Spacecraft RF Network Switch Relay Commanding and Status Monitoring;

-Spacecraft Heater Control via C\&DH Low voltage Power Converter (LVPC) $28 \mathrm{~V}$ switched power outputs;

-MAP/Launch Vehicle Separation Signal Monitoring and Distribution;

-Solar Array Deployment Activation;

- Spacecraft Telemetry Monitoring.

\section{Attitude Control Electronics (ACE)}

The MIDEX ACE subsystem (which is housed in the same enclosure as the C\&DH subsystem) acts as the primary interface between the spacecraft and the attitude control system (ACS) sensors and actuators, as well as providing an independent safehold capability to the spacecraft. The ACE implementation is a perfect example of the true value of the MIDEX architecture implementation since the type of attitude control sensors and actuators selected are highly dependent upon the specific mission and operational scenarios of a particular spacecraft. In addition, these components usually procured with varied interface options that are $\mathrm{I} / \mathrm{O}$ intensive, usually require custom interface circuitry and would not naturally fit into the MIDEX standardized 1773 bus architecture. Finally, there is the issue of distributing power from the spacecraft to the $\mathrm{ACE}$ peripheral components so that the $\mathrm{ACE}$ subsystem has the capability to turn on and off the components as required. This creates the problem of dealing with custom interfaces 
that tend to vary significantly from spacecraft to spacecraft, making it difficult to come up with a simple, standard interface approach that can be used across multiple missions with minimal redesign and non-recurring expense.

The MIDEX ACE architecture implementation addresses this problem by augmenting the Generic RSN Core design with extended input/output ( $/ / O)$ interface capabilities implemented using Field Programmable Gate Arrays (FPGAs) in order to increase flexibility for redesign between missions. The ACE $\mathrm{I} / \mathrm{O}$ design also provides as many potential I/O options as possible, allowing the spacecraft vendors to utilize commercial products and thereby keep the attitude control components cost down. The ACE I/O electronics design will be as flexible as possible to these interfaces, designed to support low-cost reconfiguration of the ACE interfaces as needed. A MIDEX common LVPC under RSN control is used to provide power switching to attitude control components.

As a result, the spacecraft to ACE subsystem interfaces comply with MIDEX architecture guidelines, consisting primarily of the 1773 fiber optic bus and power. In addition, a suite of ACE I/O cards can be developed to combine ACS component interfaces common to specific mission types on a set of ACE cards Eventually, these cards can provide a library of ACE configuration options that can be put together with minimal redesign to support specific mission requirements. The ACE configuration for the MAP mission consists of the following hardware components: the ACE RSN Card, Sensor I/O Card, and Propulsion I/O card.

ACE RSN Card- The ACE RSN card (shown in Figure 8), which is based upon the MIDEX Generic RSN Core design, provides telemetry collection and commanding for the attitude control sensors and actuators. In addition, the ACE RSN provides a hardware platform for the spacecraft independent safehold capability. In the MAP spacecraft implementation, the interfaces of spacecraft ACS sensors deemed critical for safehold operation have been designed onto the remaining card area of the ACE RSN flight card, providing a reliable, single card safehold processor that

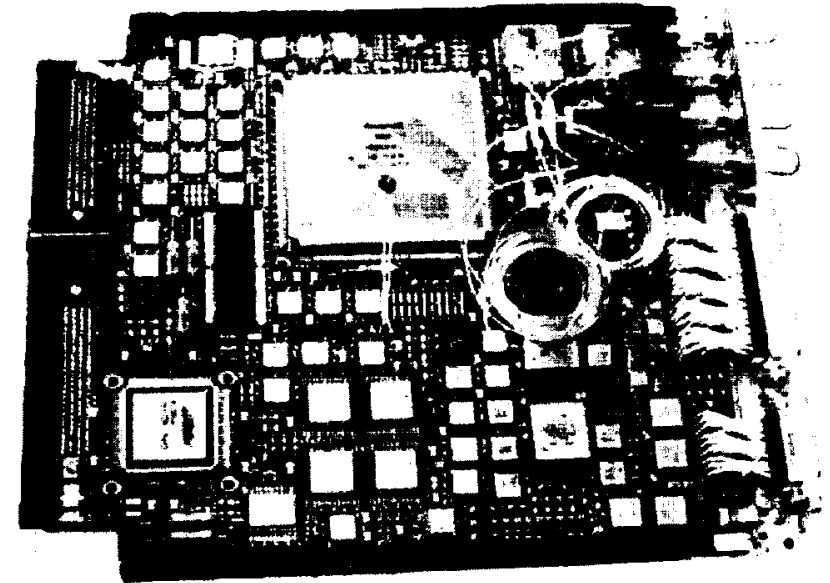

Figure 8 ACE RSN Card does not rely on any other off-card interfaces for operation. The Digital Sun Sensor (DSS) interface has also been designed into the ACE RSN card because it utilizes features already available via ESM MCM services.

Sensor //O Card- The Sensor IO card collects attitude control sensor information and transfers it to the ACE RSN for use in spacecraft attitude control calculations. In addition, the Sensor I/O card gathers spacecraft telemetry critical to spacecraft attitude control operation (such as solar array deployment information, spacecraft/launch vehicle separation signal, etc.). As mentioned previously, the actual sensor interface designs resident on this card may vary significantly based on the ACS components required for a specific mission as well as the actual ACS components manufacturers and interface options selected. The modular nature of the ACE and Sensor I/O card design supports changes in the ACS sensor contingent without massive subsystem redesign and associated NRE. In addition to these other functions, all ACS subsystem and component thermistors are monitored by the Sensor I/O card.

Propulsion I/O Card- The Propulsion I/O Cardis the sole interface for all command, control and telemetry interfcaes to the MAP propulsion system. This card provides the control capability to up to eight thrusters used in the MAP spacecraft ACS implementation. In addition, the Propulsion I/O card collects and monitors telemetry the MAP fuel tank pressure transducer, thruster temperature sensors, and is responsible for commanding and telemetry for the fuel tank isolation valve Depending on the particular ACS requirements of the spacecraft, a different card can be designed and substituted that meets other mission requirements. For example, the Propulsion I/O Card can be removed and a Magnetic Torquer $\mathrm{Bar}$ LO card can be substituted for missions requiring that type of ACS control mechanism.

\section{MIDEX Enabling Technologies}

New technology advances and infusion into spaceflight hardware has been a critical factor in realizing the MIDEX architecture concept. A few of the more important technology advancements that have enabled the MIDEX architecture implementation were the development of the Mongoose V Rad-hard 32-bit flight processor, the Essential Services Node (ESN) Multi-Chip Module (MCM), the DualRate Fiber Optic data bus, and High-Density Stacked DRAM. Each of these advancements has increased spacecraft capability while dramatically lowering overall spacecraft avionics cost, weight, and size.

\section{Mongoose V Rad-Hard 32-bit Processor}

The Mongoose V single-chip processor (Figure 9) was developed under a collaboration with GSFC, Synova Corp., and Honeywell Solid State Electronics Center (SSEC)[6]. The Mongoose $\mathrm{V}$ contains the following features:

-MIPS-compatible CPU; 
-On-chip Floating Point Unit (FPU) (compatible with ANSI/IEEE Standard 754-1985;

-On-chip instruction and data cache memory;

-Bus Interface Unit (BIU), including a programmable waitstate generator;

-Internal DRAM controller;

-Two 32-bit counter/timers;

-8-bit modified memory Error Detection and Correction (EDAC)(provides single-error correction and doubleerror detection);

-Two Asynchronous UARTs with 8251-compatible V/O;

- Commercial off-the-shelf (COTS) development tools.

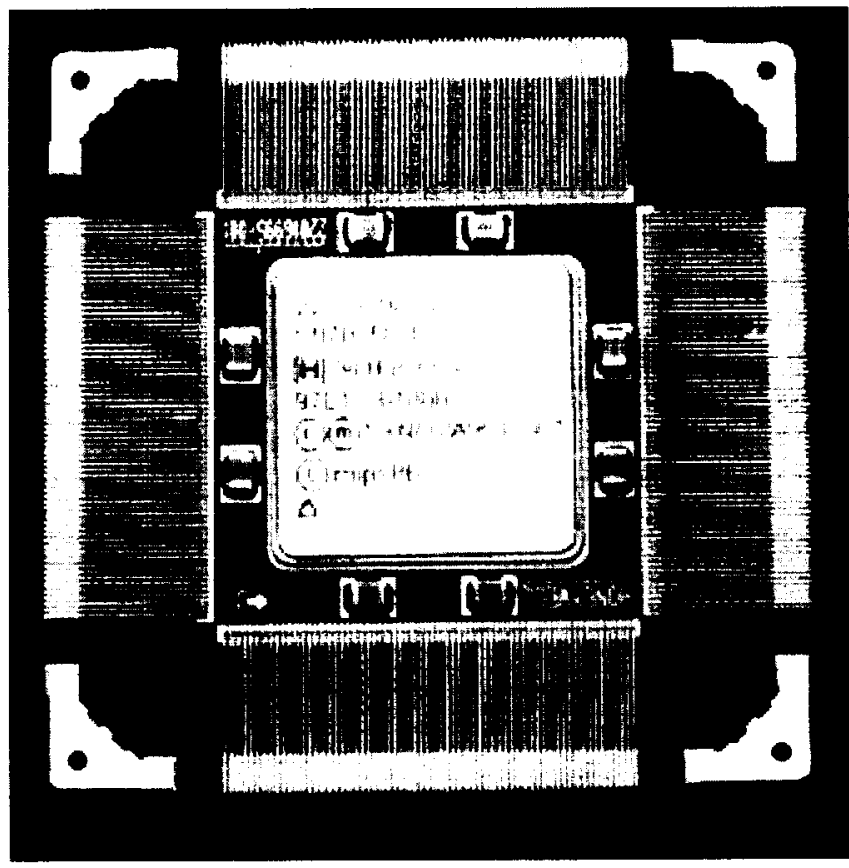

Figure 9 Mongoose V 32-bit Rad-Hard Processor

The capabilities of the Mongoose $\mathrm{V}$ processor have allowed increased capability with substantial savings in cost and weight over the XTE C\&DH subsystem, a previous inhouse GSFC spacecraft (launched 12/97) which utilized a state-of-the-art C\&DH system as part of the spacecraft avionics suite). The increased processing power of the Mongoose $\mathrm{V}$ processor allows it to perform as the Spacecraft central processor as well as the Attitude Control System processor in the MIDEX spacecraft architecture. The processor also includes an embedded Error Detection and Correction hardware capability, allowing the use of inexpensive and dense DRAM for processor program memory. The combination of both of these factors results in a capability increase, while also providing a corresponding reduction in size, weight, and a recurring cost reduction of well over $\$ 1.5 \mathrm{M}$ per $\mathrm{C} \& \mathrm{DH}$ avionics unit.

\section{Essential Services Node (ESN) Multi-Chip Module (MCM)}

The ESN (Figure 10), which was developed as part of a cooperative effort between GSFC and Honeywell Space Systems, is an analog/digital standard interface component in a MCM package that performs many spacecraft functions and is used to standardize subsystem interfaces and support the modular MIDEX architecture concept[4-5].

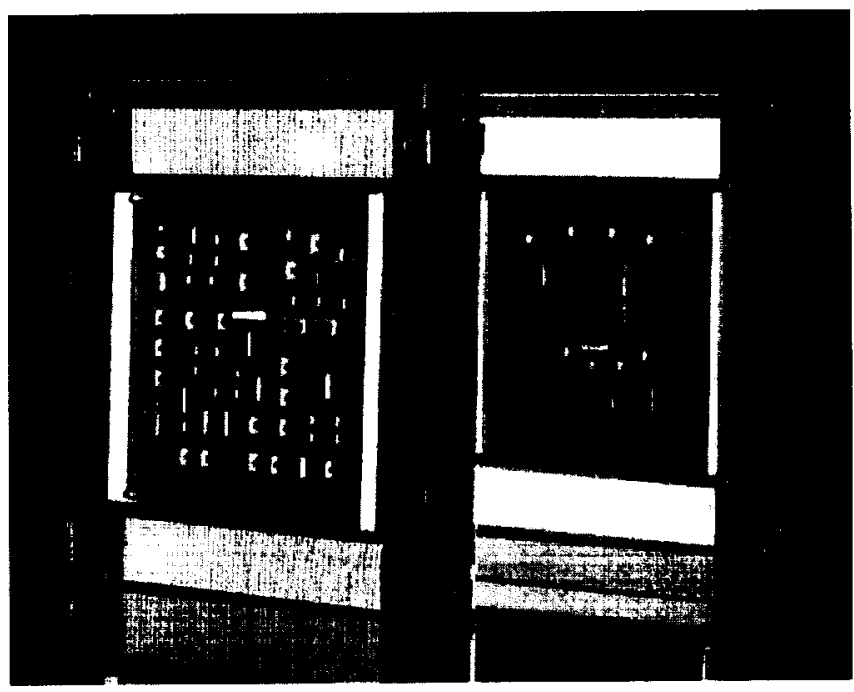

Figure 10 ESN MCM (showing analog and digital layers)

The ESN MCM contains the following features:

-MIL-STD 1553B/1773 interface

-UTMC UT69R000 microcontroller w/ 32 kwords of onboard program memory, 32 kwords of onboard data memory, 32 kwords of onboard shared memory

-Two 8251 UARTs

-8255 port device

-16-bit serial to parallel and parallel to serial converter

- 8254 counter/timer

-12-bit A/D converter

-Analog power strobing

-Sleep mode for power conservation

-Watchdog timer, Subseconds timer, Time management service

-Rad-hard component (TID $100 \mathrm{kRad-no} \mathrm{SEUs} \mathrm{or} \mathrm{latchup).}$

\section{Dual-Rate Fiber Optic Data Bus}

The use of the fiber optic bus (described previously) is a continuation of GSFC's flight fiber optic bus experience in in-house spacecraft. The use of the AS 1773 bus implementation will allow the use of higher data rates (20 Mbps) than previously available over the spacecraft 1773 bus. Not only does this increase spacecraft on-board data collection and telemetry transmission rates, but it will allow a more widespread use of the fiber optic bus for on-board data transmission in cases where higher data rate or data volume requirements prohibited the use of the 1 Mbps data bus. The elimination of discrete interfaces will, in turn, 
continue the trend towards simplifying spacecraft interfaces and decrease the complexity, time, and cost required for subsystem and spacecraft integration and test. The MAP spacecraft design utilizes the Boeing Dual-Rate Transceiver for the 1773 fiber optic bus interface implementation. A typical flight card implementation of the 1773 fiber optic components is shown in Figure 11. The fiber optic pigtails used in the transceiver design provide a tremendous degree of flexibility in selecting actual parts and connector placement of the device.

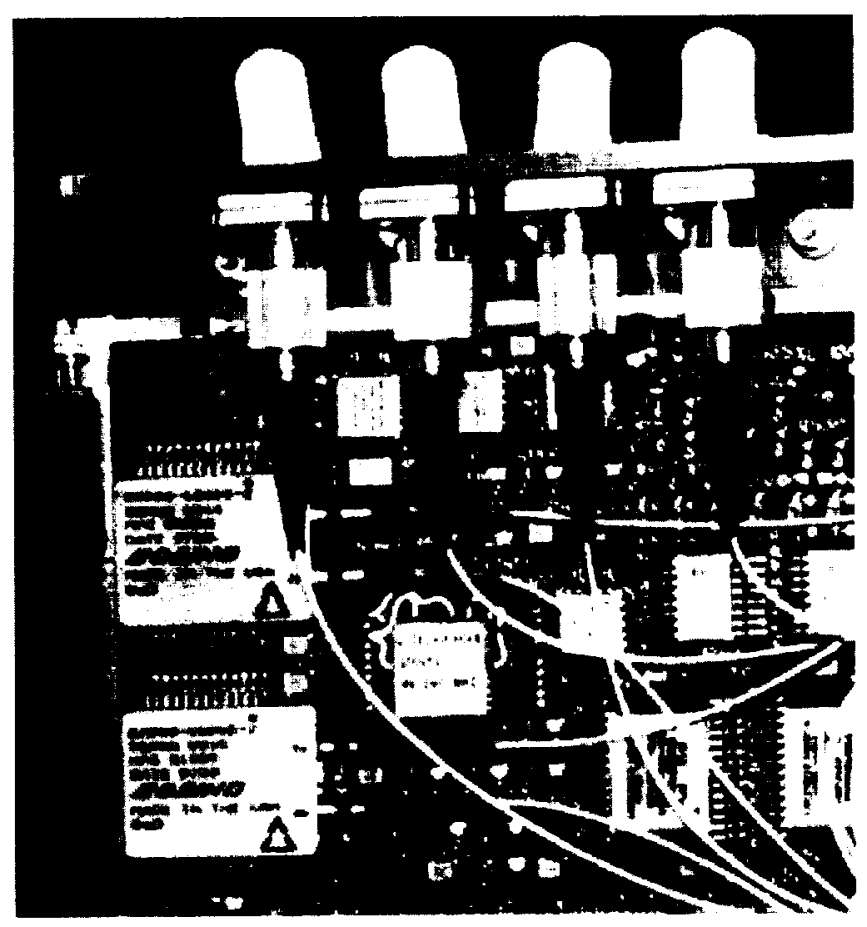

Figure 11 Typical MAP 1773 Fiber Optic Interface

High Density Stacked DRAM- The use of high-density stacked DRAM allows dramatically greater spacecraft memory storage capability at a fraction of the size, weight and cost of previous implementation approaches. For example, GSFC's most recent in-house spacecraft development efforts, XTE (launched 12/95 from KSC, FL) and TRMM (launched 11/97), both utilized similar state-ofthe art C\&DH systems in their implementations. The advent of a high-density stacked DRAM implementation (whose initial development was funded as part of a collaboration under a GSFC-led SBIR effort) was first flown in the HST SSR (part of the HST retrofit during the 1997 HST refurbishment mission). Since then, similar DRAM packaging has been implemented in the MIDEX architecture. In fact, while the TRMM SSR implementation required 14 flight cards $(\sim 8.5$ " x 10 " each) to support a 2 Gbit memory capability, the MIDEX DRAM SSR implementation actually resulted in greater memory storage capability (over 2.5 Gbits) in a flight card area of $\sim 1 / 8$ of a similarly sized card. This increased capability while decreasing size (by over 13 flight cards), decreased weight (by $\sim 30 \mathrm{lbs}$ ), and decreased recurring costs (by well over $\$ 1 \mathrm{M}$ per SSR).

\section{MIDEX ARCHITECTURE ADVANTAGES}

The advantages and benefits of the MIDEX scaleable architecture can be described in the following categories:

\section{Simplified/Standardized Interfaces}

The adoption of standardized interfaces has been shown to dramatically cut spacecraft complexity, integration and test time, and overall cost. Custom interfaces have the doubleedged impact of increased time and cost for development, maintenance and documentation, as well as increasing time and manpower required for integration and test. The MIDEX architecture emphasizes simple, standard interfaces designed to decrease development, test and integration as well as provide a simplified route to interfacing with other components on the spacecraft.

In the MIDEX architecture, the primary electrical interfaces between the subsystem components on the spacecraft are the 1773 fiber optic time multiplexed data bus and power. Discrete wire interfaces are scrupulously avoided whenever possible. Lessons learned from the X-Ray Timing Explorer (XTE), a spacecraft developed at GSFC and launched on Dec. 30, 1995, demonstrated the immense benefit of the 1773 fiber optic data bus in simplifying and shortening spacecraft integration and test, with a resultant decrease in manpower and cost [3]. Although the MIDEX architecture was designed with a AS 1773 fiber optic bus implementation (which boasts dual-rate time-multiplexed data rates of 1 and $20 \mathrm{Mbps}$ ), the initial implementation in the MAP spacecraft development program utilizes only the 1 Mbps data rate.

\section{Distributed Architecture}

In the MIDEX architecture implementation, power distribution, command decoding and telemetry collection are done in a distributed fashion at the subsystem component level rather than in a centralized fashion. This provides a number of tremendous benefits to the spacecraft development effort. Overall system level fault tolerance is increased since services and command/telemetry interfaces are distributed rather than located at a single point. The decentralized nature of the architecture also allows full testing of stand-alone subsystems apart from integration with other spacecraft components, decreasing the complexity and duration of spacecraft level I\&T.

The implementation of the distributed architecture has been enabled by the implementation of two critical technologies. The first, the 1773 fiber optic bus, permits a simplified, naturally redundant standardized command and data interface throughout the spacecraft. The second, the Essential Services Node (ESN) (described later), allows non-standard components to easily interface with the 1773 
bus in a standardized, low-cost fashion with minimal NRE. The ESN implementation also allows the spacecraft commanding and telemetry collection to be performed at the subsystem level in the decentralized fashion described above.

\section{Modular design}

All of the electronic subsystems in the MIDEX architecture (as well as the spacecraft implementation itself) follows modular, standardized design approach. Subsystem components are designed around a modular, building block approach that emphasizes standard services, cards, enclosures, and formats. This allows subsystems which can be readily configured to suit specific mission requirements. Ultimately, the MIDEX implementation approach will allow spacecraft designers to configure subsystem electronics from an existing library of cards and capabilities, tailoring the various subsystem capabilities to meet their mission's custom needs and requirements.

\section{Non-Proprietary Architecture and Development Tools}

The MIDEX development approach, as a rule, emphasizes the use of non-proprietary, commercially available industry standard formats, architectures, components, interfaces, and development tools wherever possible. This approach has the dual benefit of reducing cost as well as taking advantage of the most widely used, commercially available products on the market and making the results openly available to the commercially satellite market for development.

\section{Flexible Implementation Approach and Technology Upgrade Path}

The MIDEX architecture design and implementation approach allows users tremendous design flexibility to suit custom applications. Using the modular, standardized subsystem component designs, future implementations of spacecraft subsystems can be configured for particular mission requirements by adding or deleting cards to meet spacecraft requirements.

The modular, distributed MIDEX architecture permits new, emerging technologies be readily inserted into subsystems in a controlled manner without impacting the entire subsystem design and development. This allows a clear path to infuse new technologies and upgrade hardware without expensive and time consuming redesign issues. Previous attempts to standardize spacecraft subsystem electronics have often resulted in hardware implementations and architectures that become self-perpetuating entities, difficult to upgrade with emerging new technologies without massive redesign and accompanying high NRE. As a result, spacecraft electronics hardware either remains static and increasingly obsolete and outdated for years despite the availability of emerging new technologies, or is redesigned with each successive technology development step at tremendous manpower and expense. The MIDEX architecture implementation approach allows new technology insertion efforts to be isolated in such a manner as to avoid both of these extremes, allowing designers to easily focus on key areas where emerging technology can be targeted to further the goal of increasing spacecraft capability while lowering overall cost.

\section{Partnerships with Industry}

As part of this effort, the MIDEX implementation approach has joined in GSFC's ongoing partnerships with industry to develop components, standards and tools that further the goal of increasing capability and lowering costs. Many of the key emerging technologies that have enabled the MIDEX architecture implementation have been the result of joint development arrangements with commercial companies. For example, the rad-hard Mongoose V 32-bit RSIC processor was developed as part of a joint effort between GSFC, Synova Corp., and Honeywell SSEC; the ESN was developed in a partnership between GSFC and Honeywell Space Systems; the Boeing AS1773 fiber optic transceiver was part of a collaboration between GSFC and Boeing; and the MIDEX primary spacecraft avionics components were developed in collaboration with Litton Amecom under a NASA Space Act agreement.

\section{CONCLUSION}

The MIDEX architecture, as demonstrated in the ongoing MAP spacecraft hardware development effort, has shown itself to be a simple, more capable, and less expensive spacecraft electronics implementation approach than previous spacecraft implementations. The development costs per electronics subsystem are anticipated to be less than a third of previous missions, while still providing an increase in overall performance capability. The modular architecture has a broad applicability to a wide range of spacecraft missions, from low-end missions to those requiring full redundancy and cross-strapping of spacecraft subsystem avionics. In addition, future upgrades are already envisioned which will continue to increase capability while lowering cost. The modular nature of the MIDEX architecture allows a smooth insertion of these types of upgrades without requiring significant redesign of entire subsystems.

The MIDEX architecture hardware development for implementation in the MAP spacecraft development program is being performed as part of the in-house spacecraft development effort at GSFC. Flight unit environmental testing of all spacecraft avionics is schedule for completion in Spring 1998. This is to be followed extensive testing on the MAP "flatsat" (dedicated "flightlike" benchtop avionics testhed) and culminating with actual integration onto the MAP spacecraft in Summer 1998. 


\section{REFERENCES}

[1] Richard Day and Michael Bay, "XTE: Astronomy With Autonomy," Aerospace America, June 1996.

[2] Mark Flanegan and Ken LaBel, "Small Explorer Data System MIL-STD-1773 Fiber Optic Bus”, NASA Technical Paper 3227, June 1992.

[3] Mark M. Jarosz, John R. Kolasinski, and John Croft, "Integration and Test of the X-Ray Timing Explorer (XTE) Spacecraft Attitude Control Subsystem (ACS)," 1995 AAS Guidance and Control Conference Proceedings, February 1-5, 1995.

[4] Robert Caffrey, Michael Cuviello, Phyllis Hestnes, and Harry Shaw. "A Standard Spacecraft Data System on a Chip: NASA Goddard Space Flight Center's Essential Services Node (ESN)", 1997 IEEE Aerospace Conference Proceedings.

[5] "Essential Services Node Hardware Specification", NASA/GSFC ICD-735-2827 Rev. 2.0, August 16, 1996.

[6] "Synova Processor User's Manual, Mongoose-V Rad-Hard 32-bit Processor ASIC", Document \#MV-P-02/MV-Q-02, Rev. 1.1, July $10,1997$.

John Ruffa is a Computer Engineer in the Flight Data Systems Branch at Goddard Space Flight Center, Greenbelt, $M D$. He was previously the C\&DH Subsystem Lead on the X-Ray Timing Explorer spacecraft, which was developed in-house at GSFC and was launched from Cape Canaveral AFS, FA in December of 1995. He presently supports the Microwave Anisotropy Probe spacecraft (an in-house spacecraft development program at GSFC) as the $C \& D H, A C E$, and RF Product Team Lead. John has a $B S E E$ from the University of Maryland and an MSEE from the Johns Hopkins University.

Karen Castell is an Electrical Engineer with the Space Power Applications Branch at Goddard Space Flight Center in Greenbelt, MD. She holds a patent on a low noise, high voltage power supply design that is successfully operating on the Proportional Counter Array (PCA) instrument on the X-Ray Timing Explorer (XTE) spacecraft. She presently supports the MAP spacecraft as the Power System Lead Engineer. Karen earned her BSEE from Duke University and her MSEE from Northeastern University.

Thomas Flatley is an Electrical Engineer in the Electrical Systems Integration Branch at the Goddard Space Flight Center in Greenbelt, MD. He has worked as an electrical design engineer on the Cosmic Background Explorer (COBE), SAMPEX, XTE, and Tropical Rainfall Measurement Mission (TRMM) development teams. He presently supports the MAP spacecraft as the PSE RSN and HRSN design engineer. Tom has a BSEE from Loyola College.
Mike Lin is an Electrical Engineer in the Guidance and Control Branch at Goddard Space Flight Center in Greenbelt, MD. He was previously an Attitude Control Systems design engineer on the COBE, XTE, and TRMM spacecraft development teams. He presently supports the $M A P$ spacecraft as the ACE Lead Engineer as is also the MAP Generic RSN Lead Design Engineer. Mike has a $B S E E$ from Drexel University. 